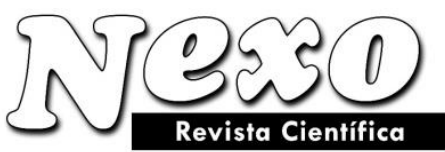

Vol. 34, No. 02, pp. 547-571/Junio 2021

\title{
Diseño de un enfriador por aire de tiro inducido con tubos aleteados para el enfriamiento de etanol
}

\section{Design of an induced draft air cooler with finned tubes for ethanol cooling}

\author{
Amaury Pérez Sánchez ${ }^{1, *}$, Yosdany Hernández González², Elizabeth Ranero González ${ }^{1}$ Eddy \\ Javier Pérez Sánchez ${ }^{3}$ \\ ${ }^{1}$ Universidad de Camagüey. Facultad de Ciencias Aplicadas. Camagüey, Cuba. \\ ${ }^{2}$ Empresa de Ingeniería y Proyectos Azucareros, UEB Centro Este. Camagüey, Cuba. \\ ${ }^{3}$ Empresa Servicios Automotores S.A., Dirección Comercial, Ciego de Ávila, Cuba. \\ *amauryps@nauta.cu
}

(recibido/received: 14-septiembre-2020; aceptado/accepted: 11-diciembre-2020)

\section{RESUMEN}

En el presente trabajo se diseñó un enfriador por aire de tiro inducido para efectuar el enfriamiento de una corriente de etanol. También se efectuó un estudio de sensibilidad para evaluar la influencia que presenta el incremento de tanto la temperatura del aire ambiente como la temperatura de entrada del etanol sobre tres parámetros de diseño. La relación porcentual entre el área proyectada por el ventilador y el área frontal $(\phi)$ tuvo un valor de 43,13\%, el cual es superior al $40 \%$ por lo que se establece que el diseño realizado es efectivo. La caída de presión total calculada para la corriente de etanol fue de 3 785,64 Pa, la cual no sobrepasa el límite máximo permisible establecido por el proceso $(10000 \mathrm{~Pa})$. La potencia calculada de cada uno de los motores acoplados a los ventiladores alcanzó un valor de $897 \mathrm{~W}$. Se sugiere operar a la menor temperatura posible del aire de entrada, y que la temperatura de alimentación del etanol al equipo se conserve en el valor establecido por el proceso $\left(80^{\circ} \mathrm{C}\right)$ para que el parámetro $\phi$ se mantenga superior al $40 \%$, y la potencia del motor no se incremente hasta alcanzar valores elevados.

Palabras claves: Caída de presión; Diseño; Enfriador por aire; Etanol; Potencia del motor.

\begin{abstract}
In the present work, an induced draft air cooler was designed to cool an ethanol stream. A sensitivity study was also carried out to evaluate the influence of increasing both the ambient air temperature and the inlet temperature of ethanol on three design parameters. The percentage ratio between the projected area of the fan and the face area of the bay $(\phi)$ had a value of $43.13 \%$, which is greater than $40 \%$, so it is established that the design carried out is effective. The total pressure drop calculated for the ethanol stream was 3,785.64 $\mathrm{Pa}$, which does not exceed the maximum allowable limit established by the process $(10,000 \mathrm{~Pa})$. The calculated power of each driver coupled to the fans reached a value of $897 \mathrm{~W}$. It is suggested to operate at the lowest inlet air temperature possible, and that the feed temperature of the ethanol to the equipment is kept at the value established by the process $\left(80^{\circ} \mathrm{C}\right)$ so that the parameter $\phi$ remains above $40 \%$, and the driver power does not increase until reaching high values.
\end{abstract}

Keywords: Pressure drop; Design; Air cooler; Ethanol; Driver power. 


\section{NOMENCLATURA}

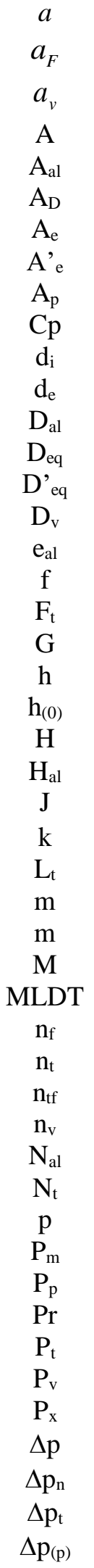

Área de flujo

$\mathrm{m}^{2}$

Área frontal

$\mathrm{m} / \mathrm{s}$

Área proyectada por los ventiladores $\quad \mathrm{m}^{2}$

Área de transferencia de calor $\quad \mathrm{m}^{2}$

Superficie de la aleta por metro de tubo $\quad \mathrm{m}^{2} / \mathrm{m}$

Superficie del tubo desnudo por metro de tubo

$\mathrm{m}^{2} / \mathrm{m}$

Ancho o amplitud del equipo

$\mathrm{m}$

Ancho o amplitud del equipo verificado

$\mathrm{m}$

Área del tubo plano por metro de tubo $\quad \mathrm{m}^{2} / \mathrm{m}$

Calor específico

Diámetro interior

$\mathrm{J} / \mathrm{kg} \cdot \mathrm{K}$

Diámetro exterior

$\mathrm{m}$

$\mathrm{m}$

Diámetro del tubo aleteado

Diámetro equivalente para la transferencia de calor

$\mathrm{m}$

diámetro equivalente por fricción $\mathrm{m}$

Diámetro del ventilador $\mathrm{m}$

Espesor de las aletas $\mathrm{m}$

Factor de fricción -

Factor de corrección de temperatura

Velocidad másica superficial

$\mathrm{kg} / \mathrm{m}^{2} . \mathrm{s}$

Coeficiente pelicular de transferencia de calor

$\mathrm{W} / \mathrm{m}^{2} . \mathrm{K}$

Coeficiente pelicular de transferencia de calor corregido

\section{Parámetro}

$\mathrm{W} / \mathrm{m}^{2} . \mathrm{K}$

Altura de las aletas

Factor de transferencia de calor

Conductividad térmica

Longitud máxima de los tubos

Caudal másico

Parámetro

Masa molar

Media Logarítmica de la Diferencia de Temperatura

Número de filas de tubos

Numero de pases por los tubos

Número de tubos por fila

Número de ventiladores

Número de aletas por metro de tubo

Número de tubos requeridos

$$
\text { Presión }
$$

Potencia de cada motor acoplado al ventilador

Perímetro proyectado

Número de Prandtl

Paso de los tubos

Presión que deberá ser desarrollada por los ventiladores

Parámetro

Caída de presión total

$\mathrm{m}$

$\mathrm{W} / \mathrm{m} . \mathrm{K}$

$\mathrm{m}$

$\mathrm{kg} / \mathrm{s}$

$-$

$\mathrm{Kg} / \mathrm{kmol}$

${ }^{\circ} \mathrm{C}$

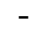

$-$

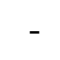

$-$

$-$

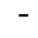

atm

W

$\mathrm{m}$

$-$

$\mathrm{m}$

$\mathrm{Pa}$

$-$

$\mathrm{Pa}$

Caída de presión debido al paso del fluido un paso hacia otro

$\mathrm{Pa}$

Caída de presión debido al paso del fluido por los tubos

$\mathrm{Pa}$

Caída de presión permisible

$\mathrm{Pa}$ 


\begin{tabular}{|c|c|c|}
\hline $\mathrm{q}$ & Caudal volumétrico & $\mathrm{m}^{3} / \mathrm{s}$ \\
\hline $\mathrm{Q}$ & Calor intercambiado & W \\
\hline $\mathrm{R}$ & Resistencia de ensuciamiento & $\mathrm{m}^{2} . \mathrm{K} / \mathrm{W}$ \\
\hline $\mathrm{R}$ & Constante de los gases ideales $=0,0821$ & atm. $\mathrm{m}^{3} / \mathrm{kmol} . \mathrm{K}$ \\
\hline $\mathrm{R}$ & Parámetro térmico & - \\
\hline $\mathrm{R}^{\prime}$ & $\begin{array}{l}\text { Resistencia de ensuciamiento corregida para la relación de diámetros } \\
\text { interno/externo }\end{array}$ & $\mathrm{m}^{2} . \mathrm{K} / \mathrm{W}$ \\
\hline $\mathrm{Re}$ & Número de Reynolds & - \\
\hline Re' & Número de Reynolds por fricción & - \\
\hline $\mathrm{S}$ & Parámetro térmico & - \\
\hline $\mathrm{S}_{\mathrm{F}}$ & Parámetro geométrico & $\mathrm{m}$ \\
\hline$S_{\mathrm{L}}$ & Parámetro geométrico & $\mathrm{m}$ \\
\hline $\mathrm{S}_{\mathrm{T}}$ & Parámetro geométrico & $\mathrm{m}$ \\
\hline $\mathrm{t}$ & Temperatura del fluido frío & ${ }^{\circ} \mathrm{C}$ \\
\hline $\mathrm{T}$ & Temperatura del fluido caliente & ${ }^{\circ} \mathrm{C}$ \\
\hline $\bar{t}$ & Temperatura media del fluido frío & ${ }^{\circ} \mathrm{C}$ \\
\hline $\bar{T}$ & Temperatura media del fluido caliente & ${ }^{\circ} \mathrm{C}$ \\
\hline$\Delta \mathrm{t}$ & Diferencia verdadera de temperatura & ${ }^{\circ} \mathrm{C}$ \\
\hline $\mathrm{U}$ & Coeficiente global de transferencia de calor & $\mathrm{W} / \mathrm{m}^{2} . \mathrm{K}$ \\
\hline $\mathrm{U}_{(\mathrm{a})}$ & Coeficiente global de transferencia de calor asumido & $\mathrm{W} / \mathrm{m}^{2} . \mathrm{K}$ \\
\hline $\mathrm{v}$ & Velocidad & $\mathrm{m} / \mathrm{s}$ \\
\hline $\mathrm{v}_{\mathrm{F}}$ & Velocidad frontal & $\mathrm{m} / \mathrm{s}$ \\
\hline $\mathrm{V}_{\mathrm{LN}}$ & Volumen libre neto & $\mathrm{m}^{3}$ \\
\hline $\mathrm{Y}$ & Parámetro & - \\
\hline $\mathrm{Z}$ & $\begin{array}{l}\text { Parámetro térmico } \\
\text { Símbolos griegos }\end{array}$ & - \\
\hline$\rho$ & Densidad & $\mathrm{kg} / \mathrm{m}^{3}$ \\
\hline$\mu$ & Viscosidad & Pa.s \\
\hline$\dot{\zeta}_{\mathrm{m}}$ & Eficiencia del motor & $\%$ \\
\hline$\zeta_{\mathrm{v}}$ & Eficiencia del ventilador & $\%$ \\
\hline$\Omega$ & Eficiencia de la aleta & - \\
\hline$\phi$ & $\begin{array}{l}\text { Relación porcentual entre el área proyectada por los ventiladores y el área } \\
\text { frontal }\end{array}$ & $\%$ \\
\hline & Subíndices & \\
\hline 1 & De entrada & \\
\hline 2 & De salida & \\
\hline et & Del etanol & \\
\hline aire & Del aire & \\
\hline
\end{tabular}

\section{INTRODUCCIÓN}

Un intercambiador de calor es un dispositivo de transferencia de calor que se emplea para la transferencia de energía térmica interna entre dos o más fluidos disponibles a diferentes temperaturas. En la mayoría de los intercambiadores de calor, los fluidos están separados por una superficie de transferencia de calor, por lo que no se mezclan entre sí (Thulukkanam, 2013).

Uno de los equipos de intercambio de calor disponibles hoy en día son los enfriadores por aire. Los enfriadores por aire son intercambiadores de calor en los cuales el calor es removido de una corriente de proceso en una operación de enfriamiento o condensación utilizando aire como medio de refrigeración (Mukherjee, 1997). La ventaja obvia que presentan estos equipos sobre los intercambiadores enfriados por 
agua es que no se necesita un circuito de agua de enfriamiento, evitando de esta manera la torre de enfriamiento, las bombas de circulación, sistemas de tratamiento de agua y las tuberías (Cao, 2010).

Se emplean en una amplia variedad de aplicaciones, fundamentalmente en refinerías, plantas petroquímicas, plantas de tratamiento de gases, estaciones de compresores, termoeléctricas, entre otras instalaciones (Boes, 2017). En alguna de estas aplicaciones se emplea el aire caliente de descarga para ayudar a calentar edificaciones y otros equipos (Amercool, 2007).

El principio de operación de un enfriador por aire es directo y simple. El fluido caliente entra por un extremo del equipo y fluye por el interior de los tubos, mientras que el aire ambiente fluye sobre y entre los tubos, los cuales típicamente presentan superficies externas aleteadas. El calor es transferido hacia el aire, el cual enfría el fluido de proceso, siendo descargado hacia la atmósfera el aire caliente (Hudson, 2007).

El costo de estas unidades es usualmente superior que el costo de los intercambiadores de calor de tubo y coraza enfriados por agua. Constituyen la única opción en aquellos lugares donde no se dispone de agua de enfriamiento, o en plantas de proceso donde los sistemas de enfriamiento por agua están operando a su máxima capacidad y resulta necesario instalar enfriadores adicionales (Cao, 2010).

La temperatura mínima bajo la cual la corriente caliente puede enfriarse se encuentra alrededor de $5{ }^{\circ} \mathrm{C}$ por encima de la temperatura del aire ambiente. La temperatura del aire varía durante el día y durante diferentes estaciones del año, por lo que resulta necesario tener información meteorológica para decidir cuál temperatura será empleada durante el diseño de estos equipos (Cao, 2010).

Existen dos tipos básicos de enfriadores por aire usualmente encontrados en las plantas petroquímicas (Boes, 2017):

- Tiro forzado: El ventilador está ubicado debajo del haz de tubos y el aire es forzado a circular a través de los tubos.

- Tiro inducido: El ventilador está localizado por encima del haz de tubos y el aire es jalado o inducido a través de los tubos.

Entre las ventajas que presentan los enfriadores por aire de tiro inducido se encuentran (Amercool, 2007) (Cao, 2010):

- Una mejor distribución del aire en la sección entera del haz de tubos.

- Menor posibilidad de recirculación del aire caliente en la sección de entrada (esto se debe a que en estas unidades el aire caliente es descargado hacia arriba a una velocidad alrededor de dos o tres veces la velocidad de entrada, debido a la relación entre el área del ventilador y la sección transversal del haz de tubos).

- Mayor capacidad en caso de que ocurra el fallo del ventilador debido al efecto de tiro natural (este efecto es muy superior en las unidades de tiro inducido que en las de tiro forzado).

- Menos afectada por la lluvia, nieve o efectos climáticos debido a que la mayoría de la cara superior del haz de tubos está cubierta.

- Accesibilidad más fácil al haz de tubos para efectuar su reemplazo.

Las principales desventajas que presentan los enfriadores por aire de tiro inducido están: 
- Mayor consumo de energía, especialmente si el incremento de la temperatura del aire es importante.

- La temperatura de salida del aire debe limitarse hasta los $90{ }^{\circ} \mathrm{C}$ para evitar daño potencial de los componentes del ventilador debido a las altas temperaturas.

- El mantenimiento del ventilador es más difícil y tiene que realizarse en el aire caliente generado por el efecto de la convección natural.

En la Figura 1 se muestra un esquema de un enfriador por aire de tiro inducido, con sus principales componentes.

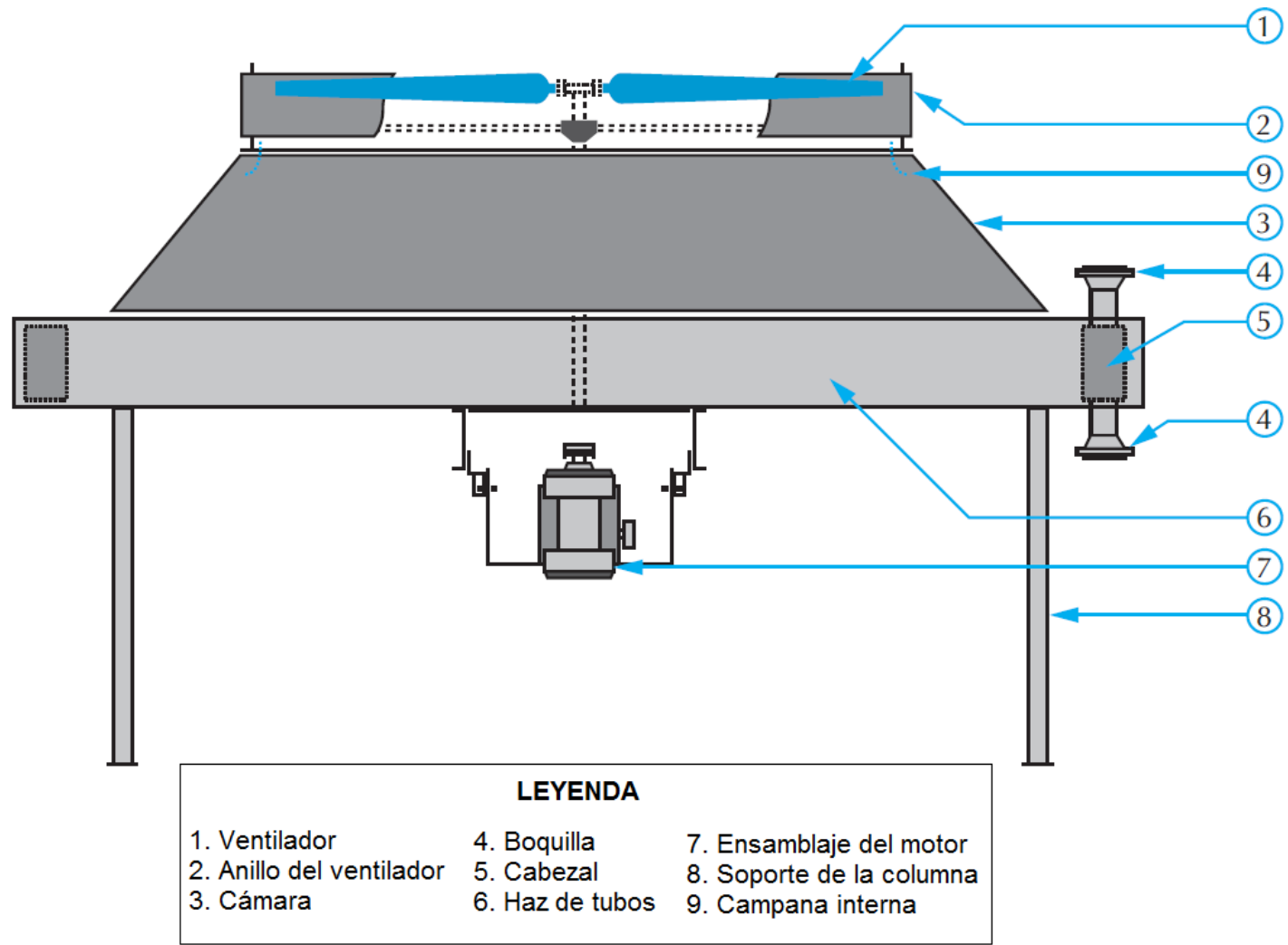

Fuente: adaptado de (Hudson, 2007)

Figura 1. Esquema de un enfriador por aire de tiro inducido.

Un enfriador por aire puede ser tan pequeño como el radiador de un auto o los suficientemente grande para cubrir varios acres de terreno, como es el caso de los enfriadores de aire empleados en grandes termoeléctricas donde el agua no está disponible (Amercool, 2007).

Hasta la fecha, son varios los autores, empresas e instituciones que han realizado estudios relacionados con los enfriadores por aire, específicamente en cuanto al diseño (Mukherjee, 1997) (Kröger, 2004) (Cao, 2010) (Yousif \& Hussain, 2012), evaluación (Elmetenani et al., 2011) (Lu et al., 2017) (Mustafa \& Jasim, 2018) y su operación o rendimiento (Giammaruti, 2004) (GPSA, 2004) (Hudson, 2007) (Amercool, 2007) (Boes, 2017) (Alfa Laval, 2018).

En el presente trabajo se realiza el diseño de un enfriador por aire de tiro inducido equipado con tubos aleteados, para efectuar el enfriamiento de una corriente de etanol desde 80 hasta $45^{\circ} \mathrm{C}$, empleando para 
ello aire ambiente a $25^{\circ} \mathrm{C}$. También se efectúa un estudio de sensibilidad donde se evalúa la influencia que presenta el incremento de tanto la temperatura del aire ambiente como la temperatura de entrada del etanol sobre tres parámetros de importancia: 1) Relación porcentual entre el área proyectada por los ventiladores y el área frontal $(\phi) ; 2)$ Caída de presión del etanol $\left(\Delta \mathrm{p}_{\mathrm{et}}\right)$; y 3 ) Potencia del motor acoplado a cada ventilador $\left(\mathrm{P}_{\mathrm{m}}\right)$. Lo anterior se realiza para observar la tendencia que presenta el incremento de ambas temperaturas sobre estos tres parámetros de diseño, y determinar así a partir de que valores de ambas temperaturas comienza a ser inefectivo el diseño del equipo.

\section{MATERIALES Y MÉTODOS}

\subsection{Definición del problema}

Se necesita diseñar un enfriador por aire de tiro inducido para enfriar $15000 \mathrm{~kg} / \mathrm{h}(4,17 \mathrm{~kg} / \mathrm{s}) \mathrm{de}$ etanol desde $80{ }^{\circ} \mathrm{C}$ hasta $45^{\circ} \mathrm{C}$, utilizando aire a $25^{\circ} \mathrm{C}$. Los tubos tendrán arreglo triangular con un paso de 60,32 $\mathrm{mm}$, una longitud de $5 \mathrm{~m}$ y un diámetro interior y exterior de $19,3 \mathrm{~mm}$ y $25,4 \mathrm{~mm}$, respectivamente. Los tubos están equipados con aletas de aluminio con una altura de $15,875 \mathrm{~mm}$, un espesor de $0,381 \mathrm{~mm}$, y poseerán 394 aletas por metro. Se permite una caída de presión de $10000 \mathrm{~Pa}$ para el etanol. Se emplearán dos ventiladores con un diámetro de $1,5 \mathrm{~m}$ y una eficiencia de $75 \%$, mientras que la eficiencia del motor será de $95 \%$. El valor del parámetro $\mathrm{S}_{\mathrm{F}}$ será $0,05223 \mathrm{~m}$ y el equipo tendrá dos pases por los tubos. La unidad será instalada a nivel del mar (presión atmosférica de $1 \mathrm{~atm}$ ).

\subsection{Metodología de cálculo a utilizar}

Para efectuar el diseño del enfriador por aire se emplearon correlaciones y ecuaciones publicadas fundamentalmente en (GPSA, 2004) (Cao, 2010), con las cuales se determina como parámetro fundamental la relación porcentual existente entre el área proyectada por los ventiladores y el área frontal, la cual debe ser mayor del $40 \%$ para que el diseño sea efectivo. También se calcula como parámetros importantes la caída de presión de la corriente caliente (etanol), y la potencia necesaria del motor acoplado a cada uno de los ventiladores. Según la metodología empleada, primeramente se efectúa un diseño preliminar, luego se realiza un diseño detallado, posteriormente se determinan las caídas de presión de cada corriente de fluido, y por último se determina la potencia de cada motor acoplado a los ventiladores.

\section{Diseño preliminar}

Paso 1: Definición de los datos iniciales disponibles:

- Temperatura de entrada y salida del etanol $\left(\mathrm{T}_{1}\right.$ y $\left.\mathrm{T}_{2}\right)$.

- Temperatura de entrada del aire $\left(\mathrm{t}_{1}\right)$

- Caudal másico del etanol $\left(\mathrm{m}_{\mathrm{et}}\right)$

- Arreglo de los tubos.

- Paso de los tubos $\left(\mathrm{P}_{\mathrm{t}}\right)$

- Longitud máxima de los tubos $\left(\mathrm{L}_{\mathrm{t}}\right)$

- Diámetro interior y exterior de los tubos $\left(\mathrm{d}_{\mathrm{i}} \mathrm{y} \mathrm{d}_{\mathrm{e}}\right)$

- Altura de las aletas $\left(\mathrm{H}_{\mathrm{al}}\right)$

- Espesor de las aletas $\left(\mathrm{e}_{\mathrm{al}}\right)$

- Número de aletas por metro de tubo $\left(\mathrm{N}_{\mathrm{al}}\right)$

- Caída de presión permisible para el etanol $\left(\Delta \mathrm{p}_{(\mathrm{p}) \mathrm{et}}\right)$

- Número de ventiladores $\left(\mathrm{n}_{\mathrm{v}}\right)$ 
- Diámetro del ventilador $\left(\mathrm{D}_{\mathrm{v}}\right)$

- Eficiencia del ventilador $\left(\zeta_{\mathrm{v}}\right)$

- Eficiencia del motor $\left(\zeta_{\mathrm{m}}\right)$

- Parámetro $S_{\mathrm{F}}$

- Número de pases por los tubos $\left(\mathrm{n}_{\mathrm{t}}\right)$.

En la Figura 2 se expone la nomenclatura utilizada tanto en los datos iniciales como en los cálculos posteriores, para efectuar el diseño del enfriador por aire.
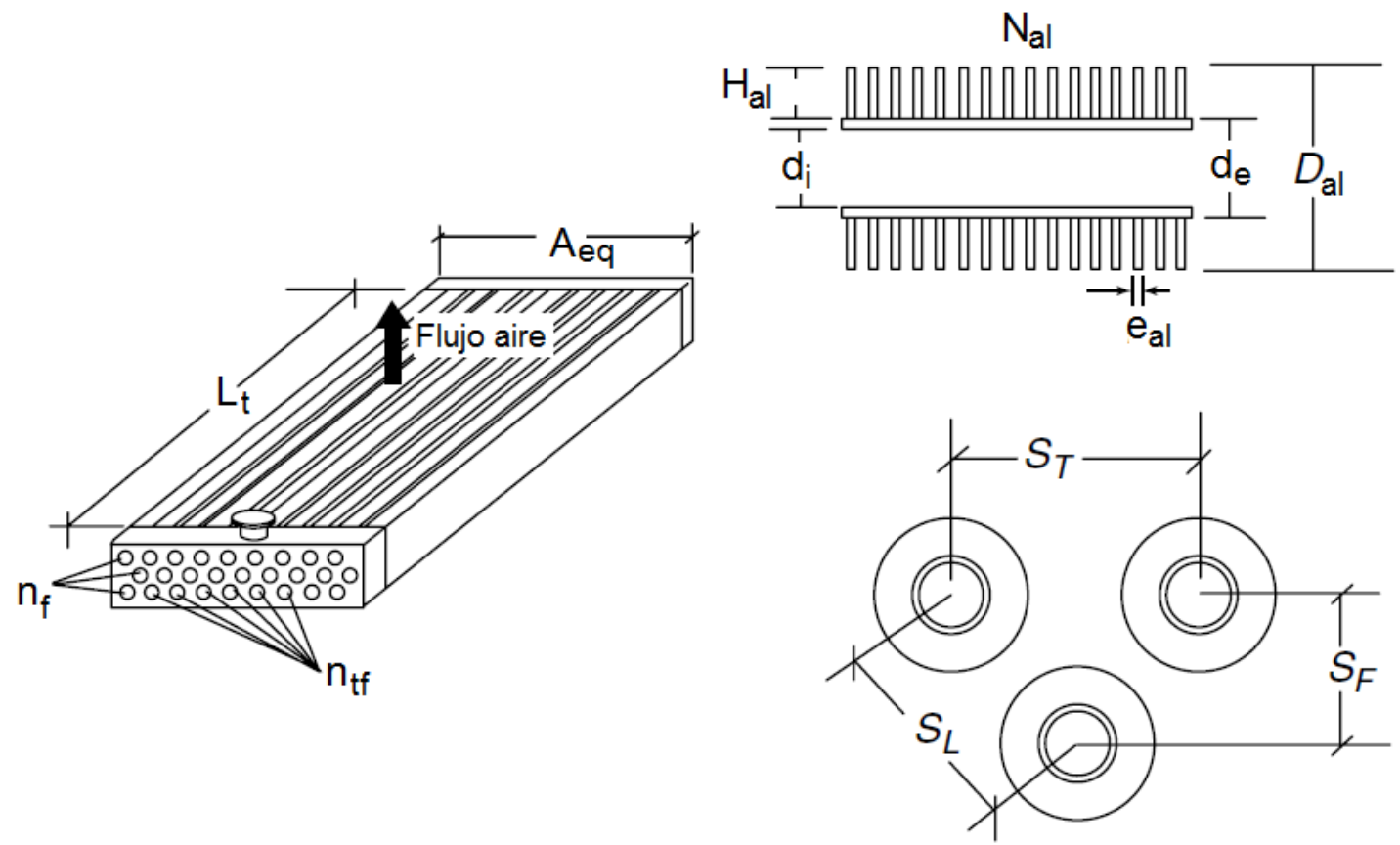

Figura 2. Nomenclatura utilizada para efectuar el diseño del enfriador por aire Fuente: adaptado de (Cao, 2010)

Paso 2. Determinación de la temperatura media del etanol $(\bar{T})$ :

$$
\bar{T}=\frac{T_{1}+T_{2}}{2}
$$

Paso 3. Búsqueda de las siguientes propiedades físicas del etanol a la temperatura media determinada en el Paso 2.

Tabla 1. Propiedades físicas requeridas del etanol a la temperatura media calculada en el Paso 2.

\begin{tabular}{lcc}
\hline Propiedad & Símbolo & Unidades \\
\hline Densidad & $\rho_{\mathrm{et}}$ & $\mathrm{kg} / \mathrm{m}^{3}$ \\
Viscosidad & $\mu_{\mathrm{et}}$ & $\mathrm{Pa} . \mathrm{s}$ \\
Conductividad térmica & $\mathrm{k}_{\mathrm{et}}$ & $\mathrm{W} / \mathrm{m} . \mathrm{K}$ \\
Calor específico & $\mathrm{Cp}_{\mathrm{et}}$ & $\mathrm{J} / \mathrm{kg} \cdot \mathrm{K}$ \\
Resistencia de ensuciamiento & $\mathrm{R}_{\mathrm{et}}$ & $\mathrm{m}{ }^{2} \cdot \mathrm{K} / \mathrm{W}$ \\
\hline Fuente: Elaboración propia & &
\end{tabular}


Paso 4. Búsqueda de las siguientes propiedades físicas del aire a la temperatura de entrada al equipo $\left(\mathrm{t}_{1}\right)$ :

Tabla 2. Propiedades físicas requeridas del aire a la temperatura de entrada al equipo.

\begin{tabular}{lcc}
\hline Propiedad & Símbolo & Unidades \\
\hline Viscosidad & $\mu_{\text {aire }}$ & Pa.s \\
Conductividad térmica & $\mathrm{k}_{\text {aire }}$ & $\mathrm{W} / \mathrm{m} . \mathrm{K}$ \\
Calor específico & Cpaire $_{\text {aire }}$ & $\mathrm{J} / \mathrm{kg} . \mathrm{K}$ \\
\hline Fuente: Elaboración propia & &
\end{tabular}

Fuente: Elaboración propia

Paso 5. Cálculo de la carga de calor o calor intercambiado (Q):

$$
Q=m_{e t} \cdot C p_{e t} \cdot\left(T_{1}-T_{2}\right)
$$

Paso 6. Suposición del coeficiente global de transferencia de calor $\left[\mathrm{U}_{(\mathrm{a})}\right]$

Paso 7. Cálculo del parámetro Z:

$$
Z=\frac{T_{1}-T_{2}}{T_{1}-t_{1}}
$$

Paso 8. Determinación del número de filas de los tubos $\left(\mathrm{n}_{\mathrm{f}}\right)$ en función del parámetro $\mathrm{Z}$ determinado en el Paso 7.

Paso 9. Determinación de la velocidad frontal $\left(\mathrm{v}_{\mathrm{F}}\right)$ en función del parámetro $\mathrm{Z}$ determinado en el Paso 7.

Paso 10. Suposición de la temperatura de salida del aire [ $\left.\mathrm{t}_{2(\mathrm{a})}\right]$.

Paso 11. Determinación del caudal de aire $\left(\mathrm{m}_{\text {aire }}\right)$ :

$$
m_{\text {aire }}=\frac{Q}{C p_{\text {aire }} \cdot\left(t_{2(a)}-t_{1}\right)}
$$

Paso 12. Cálculo de la densidad del aire a la temperatura de entrada $\left[\rho_{\text {aire(1) }}\right]$ :

$$
\rho_{\text {aire (1) }}=\frac{M_{\text {aire }} \cdot p_{\text {aire }}}{R \cdot t_{1}}
$$

Dónde:

- $\mathrm{M}_{\text {aire }}=29 \mathrm{~kg} / \mathrm{kmol}$

- $\mathrm{p}_{\text {aire }}=1 \mathrm{~atm}$

- $\mathrm{R}$ - Constante de los gases ideales $=0,0821 \mathrm{~atm} \cdot \mathrm{m}^{3} / \mathrm{kmol} . \mathrm{K}$

- $\mathrm{t}_{1}=298,15 \mathrm{~K}$

Paso 13. Cálculo del área frontal $\left(a_{F}\right)$ : 


$$
a_{F}=\frac{m_{\text {aire }}}{v_{F} \cdot \rho_{\text {aire }(1)}}
$$

Paso 14. Cálculo del ancho o amplitud del equipo $\left(\mathrm{A}_{\mathrm{e}}\right)$ :

$$
A_{e}=\frac{a_{F}}{L_{t}}
$$

Paso 15. Cálculo de la Media Logarítmica de la Diferencia de Temperatura (MLDT):

$$
M L D T=\frac{\left(T_{1}-t_{2(a)}\right)-\left(T_{2}-t_{1}\right)}{\ln \frac{\left(T_{1}-t_{2(a)}\right)}{\left(T_{2}-t_{1}\right)}}
$$

Paso 16. Área de transferencia de calor (A):

$$
A=\frac{Q}{U_{(a)} \cdot M L D T}
$$

Paso 17. Cálculo del número de tubos requeridos $\left(\mathrm{N}_{\mathrm{t}}\right)$ :

$$
N_{t}=\frac{A}{\pi \cdot d_{e} \cdot L_{t}}
$$

Paso 18. Cálculo del número de tubos por fila $\left(\mathrm{n}_{\mathrm{tf}}\right)$ :

$$
n_{t f}=\frac{N_{t}}{n_{f}}
$$

Paso 19. Verificación del ancho o amplitud del equipo $\left(A_{e}^{\prime}\right)$ :

$$
A_{e}^{\prime}=S_{T} \cdot n_{t f}
$$

Donde se considera que $\mathrm{S}_{\mathrm{T}}=\mathrm{P}_{\mathrm{t}}$

Si se cumple que $A_{e q}>A_{e q}^{\prime}$, entonces se continúa directamente con el diseño detallado. En caso contrario, se aplican los pasos $20-22$.

Paso 20. Corrección del flujo de aire ( $\left.\mathrm{m}_{\text {aire }}\right)$ :

$$
m_{\text {aire }}=v_{F} \cdot \rho_{\text {aire (1) }} \cdot A^{\prime}{ }_{e q} \cdot L_{t}
$$

Paso 21. Cálculo de la temperatura de salida del aire $\left(\mathrm{t}_{2}\right)$ : 


$$
t_{2}=t_{1}+\frac{Q}{m_{\text {aire }}^{\prime} \cdot C p_{\text {aire }}}
$$

Paso 22. Cálculo de la MLDT corregida (MLDT):

$$
M L D T=\frac{\left(T_{1}-t_{2}\right)-\left(T_{2}-t_{1}\right)}{\ln \frac{\left(T_{1}-t_{2}\right)}{\left(T_{2}-t_{1}\right)}}
$$

\section{Diseño detallado}

Paso 23. Definición de los parámetros determinados durante el diseño preliminar:

1. Número de tubos requeridos $\left(\mathrm{N}_{\mathrm{t}}\right)$

2. Número de tubos por fila $\left(\mathrm{n}_{\mathrm{tf}}\right)$

3. Número de filas de tubos $\left(\mathrm{n}_{\mathrm{f}}\right)$

4. Ancho del equipo $\left(\mathrm{A}_{\mathrm{eq}}\right)$.

5. Caudal masico de aire $\left(\mathrm{m}_{\text {aire }}\right)$.

6. Temperatura de salida del aire $\left(\mathrm{t}_{2}\right)$.

7. Media Logarítmica de la Diferencia de Temperatura (MLDT)

Paso 24. Determinación de los parámetros R y S:

$$
\begin{array}{r}
R=\frac{\left(T_{1}-T_{2}\right)}{\left(t_{2}-t_{1}\right)} \\
S=\frac{\left(t_{2}-t_{1}\right)}{\left(T_{1}-t_{1}\right)}
\end{array}
$$

Paso 25. Determinación del parámetro Px:

$$
P x=\frac{1-\left(\frac{R \cdot S-1}{S-1}\right)^{1 / N c}}{R-\left(\frac{R \cdot S-1}{S-1}\right)^{1 / N c}}
$$

Donde Nc - Número de pases por la coraza $=1$

Paso 26. Determinación del factor de corrección de temperatura $\left(\mathrm{F}_{\mathrm{t}}\right)$ :

$$
F_{t}=\frac{\sqrt{R^{2}+1}}{R-1} \cdot \frac{\ln \left[\frac{(1-P x)}{(1-R \cdot P x)}\right]}{\ln \left[\frac{\left(\frac{2}{P x}\right)-1-R+\sqrt{R^{2}+1}}{\left(\frac{2}{P x}\right)-1-R-\sqrt{R^{2}+1}}\right]}
$$


Paso 27. Determinación de la diferencia verdadera de temperatura $(\Delta t)$ :

$$
\Delta t=M L D T \cdot F_{t}
$$

Paso 28. Cálculo del área de flujo del etanol dentro de los tubos $\left(a_{e t}\right)$ :

$$
a_{e t}=\frac{N_{t} \cdot\left(\frac{\pi \cdot d_{i}^{2}}{4}\right)}{n_{t}}
$$

Paso 29. Cálculo de la velocidad del etanol dentro de los tubos ( $\left.\mathrm{v}_{\mathrm{et}}\right)$ :

$$
v_{e t}=\frac{m_{e t}}{a_{e t} \cdot \rho_{e t}}
$$

Paso 30. Cálculo del número de Reynolds del etanol $\left(\mathrm{Re}_{\mathrm{et}}\right)$ :

$$
\operatorname{Re}_{e t}=\frac{d_{i} \cdot v_{e t} \cdot \rho_{e t}}{\mu_{e t}}
$$

Paso 31. Determinación del número de Prandtl del etanol $\left(\operatorname{Pr}_{\mathrm{et}}\right)$ :

$$
\operatorname{Pr}_{e t}=\frac{C p_{e t} \cdot \mu_{e t}}{k_{e t}}
$$

Paso 32. Determinación del coeficiente pelicular de transferencia de calor del etanol $\left(\mathrm{h}_{\mathrm{et}}\right)$ :

- Régimen laminar $\left(\operatorname{Re}_{\mathrm{t}}<2100\right)$ :

$$
h_{e t}=1,86 \cdot \frac{k_{e t}}{d_{i}} \cdot\left(\frac{\operatorname{Re}_{e t} \cdot \operatorname{Pr}_{e t}}{\frac{L_{T}}{d_{i}}}\right)^{0,33}
$$

Válida para:

$0,48<\operatorname{Pr}_{\mathrm{t}}<16700$

- Régimen de transición $\left(2100 \leq \mathrm{Re}_{\mathrm{t}} \leq 10000\right)$ :

$$
h_{e t}=C p_{e t} \cdot \rho_{e t} \cdot v_{e t} \cdot 0,116 \cdot\left(\frac{\operatorname{Re}_{e t}^{0,66}-125}{\operatorname{Re}_{e t}}\right) \cdot\left[1+\left(\frac{d_{i}}{L_{t}}\right)^{0,66}\right] \cdot \operatorname{Pr}_{e t}^{-0,66}
$$

- Régimen turbulento $\left(\operatorname{Re}_{\mathrm{t}}>10000\right)$ : 


$$
h_{e t}=\frac{k_{e t}}{d_{i}} \cdot C \cdot \operatorname{Re}_{e t}^{0,8} \cdot \operatorname{Pr}_{e t}^{0,33}
$$

Dónde:

$$
\begin{aligned}
\text { C } & =0,021 \text { para gases } \\
& =0,023 \text { para líquidos no viscosos } \\
& =0,027 \text { para líquidos viscosos }
\end{aligned}
$$

\section{Válida para:}

$0,7 \leq \operatorname{Pr}_{\text {et }}<16700$

Paso 33. Corrección del coeficiente pelicular de transferencia de calor del etanol con respecto a la superficie externa del tubo $\left[h_{\text {et }(0)}\right]$ :

$$
h_{e t(0)}=h_{e t} \cdot \frac{d_{i}}{d_{e}}
$$

Paso 34. Cálculo del área del tubo plano por metro de tubo $\left(\mathrm{A}_{\mathrm{p}}\right)$ :

$$
A_{p}=\pi \cdot d_{e}
$$

Paso 35. Cálculo de la superficie del tubo desnudo por metro de tubo $\left(A_{D}\right)$ :

$$
A_{D}=A_{p} \cdot\left(1-e_{a l} \cdot N_{a l}\right)
$$

Paso 36. Determinación del diámetro del tubo aleteado $\left(\mathrm{D}_{\mathrm{al}}\right)$ :

$$
D_{a l}=d_{e}+2 \cdot H_{a l}
$$

Paso 37. Determinación de la superficie de la aleta por metro de tubo $\left(\mathrm{A}_{\mathrm{al}}\right)$ :

$$
A_{a l}=\frac{2 \cdot \pi \cdot N_{a l} \cdot\left(D_{a l}^{2}-d_{e}^{2}\right)}{4}
$$

Paso 38. Determinación del perímetro proyectado $\left(\mathrm{P}_{\mathrm{p}}\right)$ :

$$
P_{p}=2 \cdot\left(D_{a l}-d_{e}\right) \cdot N_{a l}+2 \cdot\left(1-e_{a l} \cdot N_{a l}\right)
$$

Paso 39. Cálculo del diámetro equivalente para la transferencia de calor $\left(D_{\text {eq }}\right)$ :

$$
D_{e q}=\frac{2 \cdot\left(A_{a l}+A_{D}\right)}{\pi \cdot P_{p}}
$$

Paso 40. Cálculo del área para el flujo de aire $\left(\mathrm{a}_{\text {aire }}\right)$ : 


$$
a_{\text {aire }}=A_{e} \cdot L_{t}-n_{t f} \cdot L_{t} \cdot\left[d_{e}+N_{a l} \cdot\left(D_{a l}-d_{e}\right) \cdot e_{a l}\right]
$$

Paso 41. Cálculo del número de Reynolds del aire para la transferencia de calor $\left[\operatorname{Re}_{\text {aire }}\right]$ :

$$
\operatorname{Re}_{\text {aire }}=\frac{D_{e q} \cdot m_{\text {aire }}}{a_{\text {aire }} \cdot \mu_{\text {aire }}}
$$

Paso 42. Determinación del factor J:

$$
J=0,0959 \cdot \operatorname{Re}_{\text {aire }}^{0,718}
$$

Paso 43. Cálculo del número de Prandtl del aire $\left(\operatorname{Pr}_{\text {aire }}\right)$ :

$$
\operatorname{Pr}_{\text {aire }}=\frac{C p_{\text {aire }} \cdot \mu_{\text {aire }}}{k_{\text {aire }}}
$$

Paso 44. Cálculo del coeficiente pelicular de transferencia de calor del aire para el tubo y las aletas ( $\left.\mathrm{h}_{\text {aire }}\right)$ :

$$
h_{\text {aire }}=J \cdot\left(\frac{k_{\text {aire }}}{D_{\text {eq }}}\right) \cdot \operatorname{Pr}_{\text {aire }}^{0,33}
$$

Paso 45. Cálculo del parámetro m:

$$
m=\sqrt{\frac{2 \cdot h_{\text {aire }}}{k_{a l} \cdot e_{a l}}}
$$

Donde $\mathrm{k}_{\mathrm{al}}$ - Conductividad térmica del material metálico de las aletas = $200 \mathrm{~W} / \mathrm{m} . \mathrm{K}(\mathrm{Cao}, 2010)$.

Paso 46. Determinación del parámetro H:

$$
H=\frac{D_{a l}-d_{e}}{2}
$$

Paso 47. Cálculo del parámetro Y:

$$
Y=\left(H+\frac{e_{a l}}{2}\right) \cdot\left(1+0,35 \cdot \ln \frac{D_{a l}}{d_{e}}\right)
$$

Paso 48. Cálculo de la eficiencia de la aleta $(\Omega)$ :

$$
\Omega=\frac{\tanh (m Y)}{m Y}
$$


Paso 49. Cálculo del coeficiente pelicular de transferencia de calor del aire corregido para la eficiencia de la aleta referido al área plana del tubo $\left[\mathrm{h}_{\text {aire( }(0)}\right]$ :

$$
h_{\text {aire }(0)}=\frac{h_{\text {aire }} \cdot\left(A_{D}+\Omega \cdot A_{a l}\right)}{A_{p}}
$$

Paso 50. Cálculo de la resistencia de ensuciamiento del etanol corregida para la relación de diámetros interno/externo $\left[\mathrm{R}^{\prime}\right.$ et]:

$$
R_{e t}^{\prime}=R_{e t} \cdot\left(\frac{d_{e}}{d_{i}}\right)
$$

Paso 51. Cálculo del coeficiente global de transferencia de calor (U):

$$
U=\frac{1}{\frac{1}{h_{e t(0)}}+\frac{1}{h_{\text {aire }(0)}}+R_{e t}^{\prime}}
$$

Paso 52. Cálculo del área proyectada por los ventiladores $\left(a_{v}\right)$ :

$$
a_{v}=n_{v} \cdot \frac{\pi \cdot D_{v}^{2}}{4}
$$

Paso 53. Cálculo de la relación porcentual entre el área proyectada por los ventiladores y el área frontal $(\phi)$ :

$$
\phi=\frac{a_{v}}{a_{F}} \cdot 100
$$

Se debe cumplir que $\phi \geq 40 \%$ para que el diseño sea efectivo y obtener así una distribución uniforme (Cao, 2010).

\section{Caída de presión}

- Etanol

Paso 54. Cálculo del factor de fricción del etanol $\left(\mathrm{f}_{\mathrm{et}}\right)$ :

$$
f_{e t}=1,2 \cdot\left(0,0014+\frac{0,125}{\operatorname{Re}_{e t}^{0,32}}\right)
$$

Paso 55. Determinación de la caída de presión debido al paso del etanol por los tubos del enfriador $\left(\Delta \mathrm{p}_{\mathrm{t}}\right)$ : 


$$
\Delta p_{t}=4 \cdot f_{e t} \cdot n_{t} \cdot \frac{L_{t}}{d_{i}} \cdot \rho_{e t} \cdot \frac{v_{e t}^{2}}{2}
$$

Paso 56. Cálculo de la caída de presión debido al paso del etanol un paso hacia otro $\left(\Delta \mathrm{p}_{\mathrm{n}}\right)$ :

$$
\Delta p_{n}=\frac{4 \cdot n_{t} \cdot \rho_{e t} \cdot v_{e t}^{2}}{2}
$$

Paso 57. Cálculo de la caída de presión total del etanol en el enfriador por aire $\left(\Delta \mathrm{p}_{\mathrm{et}}\right)$ :

$$
\Delta p_{e t}=\Delta p_{t}+\Delta p_{n}
$$

- Aire

Paso 58. Cálculo del volumen libre neto $\left(\mathrm{V}_{\mathrm{LN}}\right)$ :

$$
V_{L N}=A_{e} \cdot L_{t} \cdot S_{F}-n_{t f} \cdot \pi \cdot \frac{d_{e}^{2}}{4} \cdot L_{t}-N_{a l} \cdot n_{t f} \cdot L_{t} \cdot \pi \cdot\left(\frac{D_{a l}^{2}-d_{e}^{2}}{4}\right) \cdot e_{a l}
$$

Paso 59. Cálculo del diámetro equivalente por fricción ( $\mathrm{D}_{\text {eq }}$ ):

$$
D_{e q}^{\prime}=\frac{4 \cdot V_{L N}}{L_{t} \cdot n_{t f} \cdot\left(A_{a l}+A_{p}\right)}
$$

Paso 60. Cálculo del número de Reynolds del aire por fricción ( $\mathrm{Re}_{\text {aire }}$ ):

$$
\operatorname{Re}_{\text {aire }}^{\prime}=\frac{D_{e q}^{\prime} \cdot m_{\text {aire }}}{a_{\text {aire }} \cdot \mu_{\text {aire }}}
$$

Paso 61. Cálculo del factor de fricción para el aire ( $\left.\mathrm{f}_{\text {aire }}\right)$ :

$$
f_{\text {aire }}=1,276 \cdot \operatorname{Re}_{\text {aire }}^{-0,14}
$$

Paso 62. Cálculo de la temperatura media del aire en el equipo $(\bar{t})$ :

$$
\bar{t}=\frac{t_{1}+t_{2}}{2}
$$

Paso 63. Determinación de la densidad del aire a la temperatura media determinada en el paso $62\left(\bar{\rho}_{\text {aire }}\right)$ :

$$
\bar{\rho}_{\text {aire }}=\frac{M_{\text {aire }} \cdot p_{\text {aire }}}{R \cdot(\bar{t}+273,15)}
$$

Paso 64. Cálculo de la velocidad másica superficial del aire $\left(\mathrm{G}_{\text {aire }}\right)$ : 


$$
G_{\text {aire }}=\frac{m_{\text {aire }}}{a_{\text {aire }}}
$$

Paso 65. Determinación de la caída de presión total del aire a través del equipo ( $\left.\Delta \mathrm{p}_{\text {aire }}\right)$ :

$$
\Delta p_{\text {aire }}=\frac{f_{\text {aire }} \cdot G_{\text {aire }}^{2} \cdot n_{f} \cdot S_{F}}{2 \cdot \bar{\rho}_{\text {aire }} \cdot D_{\text {eq }}^{\prime}} \cdot\left(\frac{D_{e q}^{\prime}}{S_{T}}\right)^{0,4} \cdot\left(\frac{S_{L}}{S_{T}}\right)^{0,6}
$$

Donde se considera que $\mathrm{S}_{\mathrm{T}}=\mathrm{S}_{\mathrm{L}}=\mathrm{P}_{\mathrm{t}}(\mathrm{Cao}, 2010)$.

\section{Potencia del motor}

Paso 66. Determinación de la velocidad del aire en el ventilador ( $\left.v_{\text {aire }}\right)$ :

$$
v_{\text {aire }}=\frac{m_{\text {aire }}}{2 \cdot \rho_{\text {aire }(1)} \cdot \frac{\pi \cdot D_{v}^{2}}{4}}
$$

Paso 67. Determinación de la presión que deberá ser desarrollada por los ventiladores $\left(\mathrm{P}_{\mathrm{v}}\right)$ :

$$
P_{v}=\Delta p_{\text {aire }}+\frac{\rho_{\text {aire }(1)} \cdot v_{\text {aire }}^{2}}{2}
$$

Paso 68. Cálculo del caudal volumétrico de aire en cada ventilador ( $\left.\mathrm{q}_{\text {aire }}\right)$ :

$$
q_{\text {aire }}=\frac{m_{\text {aire }}}{n_{v} \cdot \rho_{\text {aire }(1)}}
$$

Paso 69. Cálculo de la potencia del motor acoplado a cada ventilador $\left(\mathrm{P}_{\mathrm{m}}\right)$ :

$$
P_{m}=\frac{q_{\text {aire }} \cdot P_{v}}{\zeta_{v} \cdot \zeta_{m}}
$$

\subsection{Estudio de sensibilidad}

Se efectuó un estudio de sensibilidad para determinar la influencia que presenta el incremento de dos variables: 1$)$ temperatura de entrada del aire ambiente $\left(\mathrm{t}_{1}\right)$ y temperatura de alimentación del etanol $\left(\mathrm{T}_{1}\right)$ sobre tres parámetros de importancia: 1) Relación porcentual entre el área proyectada por los ventiladores y el área frontal $(\phi)$; 2) Caída de presión del etanol $\left(\Delta \mathrm{p}_{\mathrm{et}}\right)$; y 3) Potencia del motor acoplado a cada ventilador $\left(\mathrm{P}_{\mathrm{m}}\right)$. En este caso la temperatura de entrada del aire ambiente se varió entre $10-35{ }^{\circ} \mathrm{C}$, mientras que la temperatura de alimentación del etanol se modificó entre $70-90^{\circ} \mathrm{C}$.

\section{RESULTADOS Y DISCUSIÓN}


A continuación, se muestran los resultados obtenidos durante el cálculo de los diferentes parámetros incluidos en la metodología de diseño del enfriador por aire de tiro inducido.

\subsection{Diseño preliminar}

Paso 1. Definición de los datos iniciales:

La Tabla 3 expone una recopilación de los datos iniciales expresados en la definición del problema (paso 1).

Tabla 3. Parámetros iniciales definidos en el paso 1.

\begin{tabular}{lccc}
\hline Parámetro & Símbolo & Valor & Unidades \\
\hline Temperatura de entrada etanol & $\mathrm{T}_{1}$ & 80 & ${ }^{\circ} \mathrm{C}$ \\
Temperatura de salida etanol & $\mathrm{T}_{2}$ & 45 & ${ }^{\circ} \mathrm{C}$ \\
Temperatura de entrada del aire & $\mathrm{t}_{1}$ & 25 & ${ }^{\circ} \mathrm{C}$ \\
Caudal másico de etanol & $\mathrm{m}_{\mathrm{et}}$ & 4,17 & $\mathrm{~kg} / \mathrm{s}$ \\
Arreglo de los tubos & - & $\mathrm{Triangular}$ & - \\
Paso de los tubos & $\mathrm{P}_{\mathrm{t}}$ & 0,06032 & $\mathrm{~m}$ \\
Longitud máxima de los tubos & $\mathrm{L}_{\mathrm{t}}$ & 5 & $\mathrm{~m}$ \\
Diámetro interior de los tubos & $\mathrm{d}_{\mathrm{i}}$ & 0,01930 & $\mathrm{~m}$ \\
Diámetro exterior de los tubos & $\mathrm{d}_{\mathrm{e}}$ & 0,02540 & $\mathrm{~m}$ \\
Altura de las aletas & $\mathrm{H}_{\mathrm{al}}$ & 0,015875 & $\mathrm{~m}$ \\
Espesor de las aletas & $\mathrm{e}_{\mathrm{al}}$ & 0,000381 & $\mathrm{~m}$ \\
Número de aletas por metro & $\mathrm{N}_{\mathrm{al}}$ & 394 & - \\
Cá́da de presión permisible para el etanol & $\mathrm{p}_{\mathrm{p} p) \text { et }}$ & 10 & $\mathrm{kPa}$ \\
Número de ventiladores & $\mathrm{n}_{\mathrm{v}}$ & 2 & - \\
Diámetro del ventilador & $\mathrm{D}_{\mathrm{v}}$ & 1,5 & $\mathrm{~m}$ \\
Eficiencia del ventilador & $\zeta_{\mathrm{v}}$ & 0,75 & - \\
Eficiencia del motor & $\zeta_{\mathrm{m}}$ & 0,95 & - \\
Parámetro S & $\mathrm{S}_{\mathrm{F}}$ & 0,05223 & $\mathrm{~m}$ \\
Número de pases por los tubos & $\mathrm{n}_{\mathrm{t}}$ & 2 & - \\
\hline Fuente: Elaboración propia & & &
\end{tabular}

Paso 2: Temperatura media del etanol:

$$
\bar{T}=\frac{T_{1}+T_{2}}{2}=\frac{80+45}{2}=62,5^{\circ} \mathrm{C}
$$

Paso 3. Propiedades físicas del etanol a la temperatura media $\bar{T}=62,5^{\circ} \mathrm{C}$.

Según (Perry \& Green, 2008), el etanol presenta los siguientes valores de las propiedades físicas a la temperatura media $(\bar{T})$ determinada en el paso anterior (Tabla 4 ).

Tabla 4. Propiedades físicas del etanol a la temperatura media $\bar{T}=62,5^{\circ} \mathrm{C}$.

\begin{tabular}{lccc}
\hline Propiedad & Símbolo & Valor & Unidades \\
\hline Densidad & $\rho_{\mathrm{et}}$ & 750,75 & $\mathrm{~kg} / \mathrm{m}^{3}$ \\
Viscosidad & $\mu_{\mathrm{et}}$ & 0,000561 & $\mathrm{Pa.s}$ \\
Conductividad térmica & $\mathrm{k}_{\mathrm{et}}$ & 0,158 & $\mathrm{~W} / \mathrm{m} . \mathrm{K}$ \\
Calor específico & $\mathrm{Cp}_{\mathrm{et}}$ & 2809,84 & $\mathrm{~J} / \mathrm{kg} . \mathrm{K}$ \\
Resistencia de ensuciamiento & $\mathrm{R}_{\mathrm{et}}$ & 0,0002 & $\mathrm{~m}^{2} . \mathrm{K} / \mathrm{W}$ \\
\hline
\end{tabular}


Fuente: Elaboración propia

Paso 4. Propiedades físicas del aire a la temperatura de entrada al equipo $\left(\mathrm{t}_{1}\right)$ :

Según (Mijeev \& Mijeeva, 1979), el aire presenta las siguientes propiedades físicas a $t_{1}=25^{\circ} \mathrm{C}$ (Tabla 5).

Tabla 5. Propiedades físicas del aire a la temperatura de entrada al enfriador $\left(\mathrm{t}_{1}=25^{\circ} \mathrm{C}\right)$.

\begin{tabular}{lccc}
\hline Propiedad & Símbolo & Valor & Unidades \\
\hline Viscosidad & $\mu_{\text {aaire }}$ & 0,0000183 & Pa.s \\
Conductividad térmica & $\mathrm{k}_{\text {aire }}$ & 0,0263 & $\mathrm{~W} / \mathrm{m} . \mathrm{K}$ \\
Calor específico & $\mathrm{Cp}_{\text {aire }}$ & 1005 & $\mathrm{~J} / \mathrm{kg} . \mathrm{K}$ \\
\hline Fuente: Elaboración propia & & &
\end{tabular}

Paso 5. Calor intercambiado (Q):

$$
Q=m_{e t} \cdot C p_{e t} \cdot\left(T_{1}-T_{2}\right)=410096,15 W
$$

Paso 6. Suposición del coeficiente global de transferencia de calor:

Según (Cao, 2010), para las condiciones de operación establecidas en este proyecto se tiene un valor del coeficiente global de transferencia de calor $\left[\mathrm{U}_{(\mathrm{a})}\right]$ de $600 \mathrm{~W} / \mathrm{m}^{2} . \mathrm{K}$.

Paso 7. Cálculo del parámetro Z:

$$
Z=\frac{T_{1}-T_{2}}{T_{1}-t_{1}}=0,636
$$

Paso 8. Número de filas de tubos $\left(\mathrm{n}_{\mathrm{f}}\right)$ en función del parámetro $\mathrm{Z}$ :

De acuerdo con $(\mathrm{Cao}, 2010)$, para un valor de $\mathrm{Z}=0,636$ se tiene un resultado del número de filas de tubos $\left(\mathrm{n}_{\mathrm{f}}\right)$ de 6.

Paso 9. Velocidad frontal $\left(\mathrm{v}_{\mathrm{F}}\right)$ en función del parámetro Z:

Conforme con (Cao, 2010), para un valor de $Z=0,636$ se tiene un resultado de $v_{F}$ de $2,8 \mathrm{~m} / \mathrm{s}$.

Paso 10. Suposición de la temperatura de salida del aire:

Se asume una temperatura de salida del aire $\left[\mathrm{t}_{2(\mathrm{a})}\right]$ de $40^{\circ} \mathrm{C}$.

La Tabla 6 describe los resultados de los parámetros determinados en los pasos 11-19.

Tabla 6. Resultados de los parámetros determinados en los pasos 11-19.

\begin{tabular}{clccc}
\hline Paso & Parámetro & Símbolo & Valor & Unidades \\
\hline 11 & Caudal de aire & $\mathrm{m}_{\text {aire }}$ & 27,20 & $\mathrm{~kg} / \mathrm{s}$ \\
12 & Densidad del aire a la temperatura de entrada & $\rho_{\text {aire }(1)}$ & 1,185 & $\mathrm{~kg} / \mathrm{m}^{3}$ \\
13 & Área frontal & $a_{F}$ & 8,19 & $\mathrm{~m}^{2}$ \\
14 & Ancho o amplitud del equipo & $\mathrm{A}_{\mathrm{e}}$ & 1,64 & $\mathrm{~m}$ \\
15 & Media logarítmica de la diferencia de temperatura & MLDT & 28,85 & ${ }^{\circ} \mathrm{C}$
\end{tabular}




\begin{tabular}{clccc}
16 & Área de transferencia de calor & $\mathrm{A}$ & 23,69 & $\mathrm{~m}^{2}$ \\
17 & Número de tubos & $\mathrm{N}_{\mathrm{t}}$ & 60 & - \\
18 & Número de tubos por fila & $\mathrm{n}_{\mathrm{tf}}$ & 10 & - \\
19 & Verificación del ancho o amplitud del equipo & $\mathrm{A}_{\mathrm{e}}^{\prime}$ & 0,60 & $\mathrm{~m}$ \\
\hline Fuente: Elaboración propia & &
\end{tabular}

Como se cumple la condición de que $A_{e}>A_{e}^{\prime}(1,64>0,60)$ entonces se continúa directamente con el diseño detallado.

\subsection{Diseño detallado}

Paso 23. Definición de los parámetros determinados durante el diseño preliminar:

1. Número de tubos requeridos $\left(\mathrm{N}_{\mathrm{t}}\right)=60$

2. Número de tubos por filla $\left(\mathrm{n}_{\mathrm{tf}}\right)=10$

3. Número de filas de tubos $\left(\mathrm{n}_{\mathrm{f}}\right)=6$

4. Ancho del equipo $\left(\mathrm{A}_{\mathrm{e}}\right)=1,64 \mathrm{~m}$

5. Caudal masico de aire $\left(\mathrm{m}_{\text {aire }}\right)=27,20 \mathrm{~kg} / \mathrm{s}$.

6. Temperatura de salida del aire $\left(\mathrm{t}_{2}\right)=40^{\circ} \mathrm{C}$

7. Media Logarítmica de la Diferencia de Temperatura $(\mathrm{MLDT})=28,85^{\circ} \mathrm{C}$.

La siguiente tabla (Tabla 7) presenta los resultados de los parámetros determinados en los pasos 24-31.

Tabla 7. Resultados de los parámetros calculados en los pasos 24-31.

\begin{tabular}{clccc}
\hline Paso & Parámetro & Símbolo & Valor & Unidades \\
\hline 24 & Parámetro R & $\mathrm{R}$ & 2,33 & - \\
& Parámetro S & $\mathrm{S}$ & 0,27 & - \\
25 & Parámetro Px & $\mathrm{Px}$ & 0,27 & - \\
26 & Factor de corrección de temperatura & $\mathrm{F}_{\mathrm{t}}$ & 0,88 & - \\
27 & Diferencia verdadera de temperatura & $\Delta \mathrm{t}$ & 25,38 & ${ }^{\circ} \mathrm{C}$ \\
28 & Área de flujo del etanol dentro de los tubos & $\mathrm{a}_{\mathrm{et}}$ & 0,0088 & $\mathrm{~m}^{2}$ \\
29 & Velocidad del etanol dentro de los tubos & $\mathrm{v}_{\mathrm{et}}$ & 0,63 & $\mathrm{~m} / \mathrm{s}$ \\
30 & Número de Reynolds del etanol & $\mathrm{Re}_{\mathrm{et}}$ & 16271,60 & - \\
31 & Número de Prandtl del etanol & $\mathrm{Pr}_{\mathrm{et}}$ & 9,98 & - \\
\hline Fuente: Elaboración propia & & &
\end{tabular}

Paso 32. Coeficiente pelicular de transferencia de calor del etanol $\left(\mathrm{h}_{\mathrm{et}}\right)$ :

Como el etanol fluye bajo régimen de flujo turbulento, ya que se cumple que $R_{e t}>10000$, entonces se hará uso de la ecuación (27). De esta manera:

$$
h_{e t}=\frac{k_{e t}}{d_{i}} \cdot 0,023 \cdot \operatorname{Re}_{e t}^{0,8} \cdot \operatorname{Pr}_{e t}^{0,33}=943,12 W / m^{2} \cdot K
$$

La Tabla 8 expone los resultados obtenidos de los parámetros incluidos en los pasos 33-53.

Tabla 8. Resultados de los parámetros calculados en los pasos 33-53.

\begin{tabular}{clccc}
\hline Paso & Parámetro & Símbolo & Valor & Unidades \\
\hline 33 & Coeficiente pelicular del transferencia de calor del & $\mathrm{h}_{\mathrm{et}(0)}$ & 716,62 & $\mathrm{~W} / \mathrm{m}^{2} . \mathrm{K}$
\end{tabular}
etanol corregido con respecto a las superficie externa 


\begin{tabular}{|c|c|c|c|c|}
\hline & del tubo & & & \\
\hline 34 & Área del tubo plano por metro de tubo & $A_{p}$ & 0,0798 & $\mathrm{~m}^{2} / \mathrm{m}$ \\
\hline 35 & Superficie del tubo desnudo por metro de tubo & $A_{D}$ & 0,0678 & $\mathrm{~m}^{2} / \mathrm{m}$ \\
\hline 36 & Diámetro del tubo aleteado & $\mathrm{D}_{\mathrm{al}}$ & 0,0572 & $\mathrm{~m}$ \\
\hline 37 & Superficie de la aleta & $\mathrm{A}_{\mathrm{al}}$ & 1,6237 & $\mathrm{~m}^{2} / \mathrm{m}$ \\
\hline 38 & Perímetro proyectado & $\mathrm{P}_{\mathrm{p}}$ & 26,76 & $\mathrm{~m}$ \\
\hline 39 & Diámetro equivalente para la transferencia de calor & $\mathrm{D}_{\mathrm{eq}}$ & 0,0403 & $\mathrm{~m}$ \\
\hline 40 & Área para el flujo de aire & $a_{\text {aire }}$ & 6,69 & $\mathrm{~m}^{2}$ \\
\hline 41 & $\begin{array}{l}\text { Número de Reynolds del aire para la transferencia } \\
\text { de calor }\end{array}$ & $\operatorname{Re}_{\text {aire }}$ & 8953,58 & - \\
\hline 42 & Factor J & $\mathrm{J}$ & 65,97 & - \\
\hline 43 & Número de Prandtl del aire & $\operatorname{Pr}_{\text {aire }}$ & 0,699 & - \\
\hline 44 & $\begin{array}{l}\text { Coeficiente pelicular de transferencia de calor del } \\
\text { aire para el tubo y las aletas }\end{array}$ & $\mathrm{h}_{\text {aire }}$ & 38,25 & $\mathrm{~W} / \mathrm{m}^{2} . \mathrm{K}$ \\
\hline 45 & Parámetro m & $\mathrm{m}$ & 31,68 & - \\
\hline 46 & Parámetro $\mathrm{H}$ & $\mathrm{H}$ & 0,0159 & - \\
\hline 47 & Parámetro Y & $\mathrm{Y}$ & 0,0207 & - \\
\hline 48 & Eficiencia de la aleta & $\Omega$ & 0,8776 & - \\
\hline 49 & $\begin{array}{l}\text { Coeficiente pelicular de transferencia de calor del } \\
\text { aire corregido para la eficiencia de la aleta y referido } \\
\text { al área plana del tubo }\end{array}$ & $\mathrm{h}_{\text {aire(0) }}$ & 715,51 & $\mathrm{~W} / \mathrm{m}^{2} . \mathrm{K}$ \\
\hline 50 & $\begin{array}{l}\text { Resistencia de ensuciamiento del etanol corregida } \\
\text { para la relación de diámetros interno/externo }\end{array}$ & $\mathrm{R}_{\text {et }}^{\prime}$ & 0,00026 & $\mathrm{~m}^{2} . \mathrm{K} / \mathrm{W}$ \\
\hline 51 & Coeficiente global de transferencia de calor & U & 327,59 & $\mathrm{~W} / \mathrm{m}^{2} . \mathrm{K}$ \\
\hline 52 & Área proyectada por los ventiladores & $a_{v}$ & 3,53 & $\mathrm{~m}^{2}$ \\
\hline 53 & $\begin{array}{l}\text { Relación porcentual entre el área proyectada por los } \\
\text { ventiladores y el área frontal }\end{array}$ & $\phi$ & 43,13 & $\%$ \\
\hline
\end{tabular}

El equipo tendrá un ancho de 1,64 m, una cantidad de tubos igual a 60, un número de filas de tubos de 6, y un número de tubos por fila de 10. El coeficiente pelicular de transferencia de calor del etanol alcanzó un valor de $943,12 \mathrm{~W} / \mathrm{m}^{2}$.K. Se obtuvo una eficiencia de la aleta de 0,8776 , un coeficiente pelicular de transferencia de calor para el aire, corregido para la eficiencia de la aleta y referido al área plana del tubo, de $715,51 \mathrm{~W} / \mathrm{m}^{2} . \mathrm{K}$, y un coeficiente global de transferencia de calor calculado de $327,59 \mathrm{~W} / \mathrm{m}^{2} . \mathrm{K}$, el cual es un 45,4 \% menor que el asumido en el Paso $6\left(600 \mathrm{~W} / \mathrm{m}^{2} . \mathrm{K}\right)$. Por último, el parámetro relación porcentual entre el área proyectada por los ventiladores y el área frontal $(\phi)$ tuvo un valor de 43,13\%, el cual es superior al $40 \%$ por lo que se concluye que el diseño es efectivo, obteniéndose así una distribución uniforme en el enfriador (Cao, 2010).

\subsection{Caída de presión}

La Tabla 9 describe los valores de los parámetros determinados en los pasos 54-65, relacionados con la determinación de las caídas de presión de tanto el etanol como el aire.

Tabla 9. Resultados de los parámetros determinados en los pasos 54-65.

\begin{tabular}{|c|c|c|c|c|}
\hline Paso & Parámetro & Símbolo & Valor & Unidades \\
\hline \multicolumn{5}{|c|}{ Etanol } \\
\hline 54 & Factor de fricción del etanol en los tubos & $f_{e t}$ & 0,0084 & - \\
\hline 55 & $\begin{array}{l}\text { Caída de presión debido al paso del etanol por los } \\
\text { tubos del enfriador }\end{array}$ & $\Delta \mathrm{p}_{\mathrm{t}}$ & 2593,75 & $\mathrm{~Pa}$ \\
\hline 56 & caída de presión Debido al paso del etanol un paso & $\Delta \mathrm{p}_{\mathrm{n}}$ & 1191,89 & $\mathrm{~Pa}$ \\
\hline
\end{tabular}


Caída de presión total del etanol en el enfriador por aire

\begin{tabular}{cl} 
& \\
58 & Volumen libre neto \\
59 & Diámetro equivalente por fricción \\
60 & Número de Reynolds del aire por fricción \\
61 & Factor de fricción para el aire \\
62 & Temperatura media del aire en el equipo \\
63 & Densidad del aire a la temperatura media \\
64 & Velocidad másica superficial del aire \\
65 & Caída de presión del aire a través del equipo \\
\hline Fuente: Elaboración propia
\end{tabular}

$\Delta \mathrm{p}_{\mathrm{et}} \quad 3785,64 \quad \mathrm{~Pa}$

\begin{tabular}{ccc} 
& & \\
$\mathrm{V}_{\mathrm{LN}}$ & 0,3875 & $\mathrm{~m}^{3}$ \\
$\mathrm{D}_{\text {eq }}$ & 0,0182 & $\mathrm{~m}$ \\
$\mathrm{Re}_{\text {aire }}$ & 4044,44 & - \\
$\mathrm{f}_{\text {aire }}$ & 0,399 & - \\
$\bar{t}$ & 32,50 & ${ }^{\circ} \mathrm{C}$ \\
$\bar{\rho}_{\text {aire }}$ & 1,156 & $\mathrm{~kg} / \mathrm{m}^{3}$ \\
$\mathrm{G}_{\text {aire }}$ & 4,07 & $\mathrm{~kg} / \mathrm{m}^{2} . \mathrm{s}$ \\
$\Delta \mathrm{p}_{\text {aire }}$ & 30,48 & $\mathrm{~Pa}$ \\
\hline
\end{tabular}

La caída de presión total del etanol por los tubos del enfriador alcanzó un valor de 3 785,64 Pa, el cual es inferior a la caída de presión permisible establecida para esta corriente (10 $000 \mathrm{~Pa})$. Por su parte, el aire presentó una caída de presión de 30,48 Pa.

\subsection{Potencia del motor}

La Tabla 10 presenta los resultados de los parámetros determinados en los pasos 66-69, correspondientes a la determinación de la potencia que deberá consumir cada motor acoplado a cada uno de los ventiladores.

Tabla 10. Resultados de los parámetros determinados en los pasos 66-69.

\begin{tabular}{|c|c|c|c|c|}
\hline Paso & Parámetro & Símbolo & Valor & Unidades \\
\hline 66 & Velocidad del aire en el ventilador & Vaire & 6,49 & $\mathrm{~m} / \mathrm{s}$ \\
\hline 67 & $\begin{array}{l}\text { Presión que deberá ser desarrollada por los } \\
\text { ventiladores }\end{array}$ & $\mathrm{P}_{\mathrm{v}}$ & 55,44 & $\mathrm{~Pa}$ \\
\hline 68 & Caudal volumétrico de aire en los ventiladores & qaire & 11,48 & $\mathrm{~m}^{3} / \mathrm{s}$ \\
\hline 69 & Potencia del motor acoplado a cada ventilador & $P_{m}$ & 897 & $\mathrm{~W}$ \\
\hline
\end{tabular}

De acuerdo con los resultados mostrados en la Tabla 8, cada uno de los dos motores utilizados para hacer funcionar a los dos ventiladores consume una potencia de $897 \mathrm{~W}$. También se puede observar que los dos ventiladores son capaces de procesar $11,48 \mathrm{~m}^{3} / \mathrm{s}$ de aire.

\subsection{Estudio de sensibilidad}

La Figura 3 muestra los resultados obtenidos al determinar la influencia que presenta el incremento de la temperatura de entrada del aire ambiente $\left(t_{1}\right)$ sobre los tres parámetros de diseño evaluados, esto es: 1$)$ Relación porcentual entre el área proyectada por los ventiladores y el área frontal $(\phi) ; 2)$ Caída de presión total del etanol $\left(\Delta \mathrm{p}_{\mathrm{et}}\right)$; y 3$)$ Potencia del motor acoplado a cada ventilador $\left(\mathrm{P}_{\mathrm{m}}\right)$. La Figura 4 expresa, por su parte, el efecto que presenta un incremento de la temperatura de alimentación del etanol $\left(\mathrm{T}_{1}\right)$ sobre los tres parámetros de diseño descritos con anterioridad. 


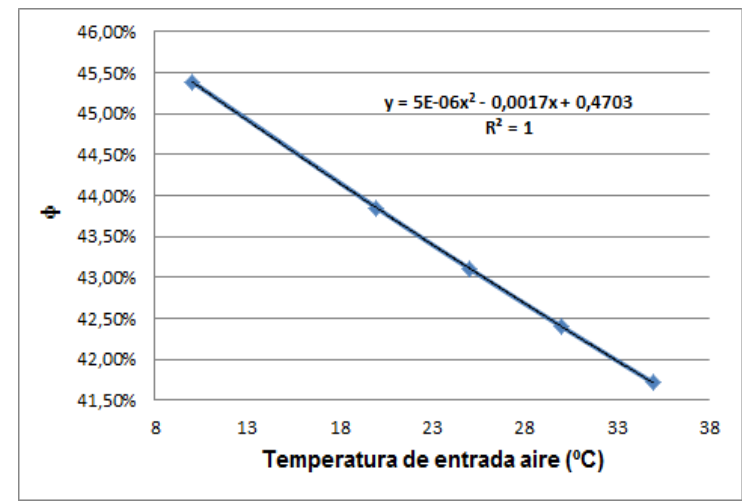

(a)

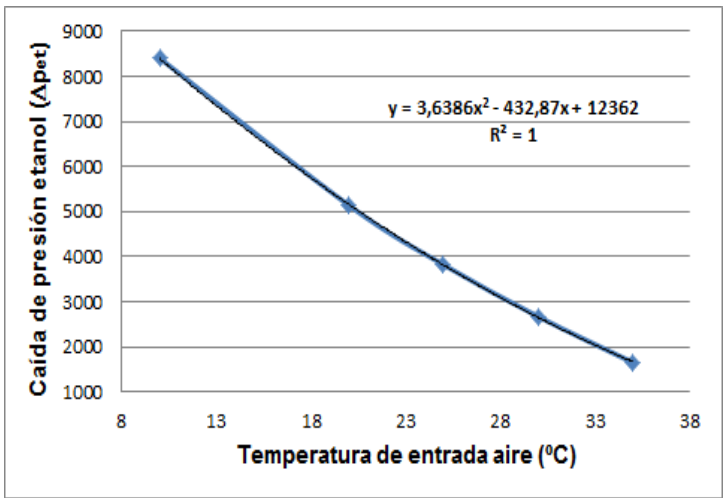

(b)

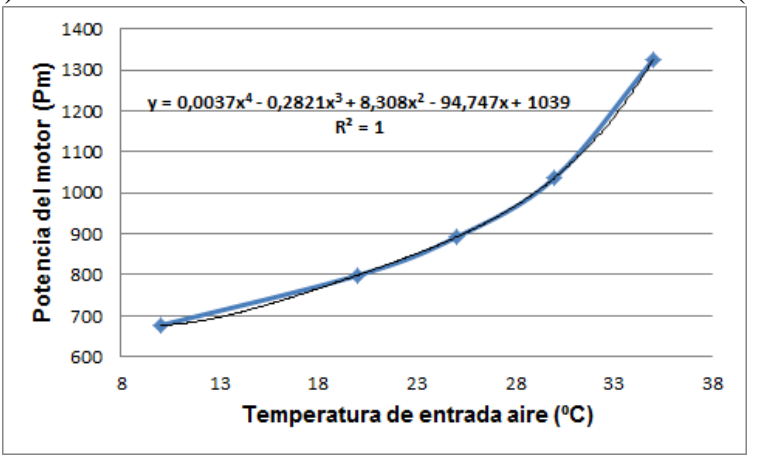

(c)

Figura 3. Influencia del incremento de la temperatura de entrada del aire ambiente sobre tres parámetros de diseño del equipo. (a) Relación porcentual entre el área proyectada por los ventiladores y el área frontal $(\phi)$. (b) Caída de presión total del etanol ( $\left.\Delta \mathrm{p}_{\mathrm{et}}\right)$. (c) Potencia del motor acoplado a cada ventilador $\left(\mathrm{P}_{\mathrm{m}}\right)$. Fuente: Elaboración propia

Según se puede observar en la Figura 3 , tanto el parámetro $\phi$ como la caída de presión del etanol disminuyen a medida que aumenta la temperatura de entrada del aire ambiente $\left(\mathrm{t}_{1}\right)$, mientras que la potencia del motor se incrementa. Vale destacar que aún a una temperatura de $35^{\circ} \mathrm{C}$ el valor del parámetro $\phi$ se mantiene por encima del $40 \%$, lo cual denota que el aire puede alcanzar esta temperatura y el diseño del equipo puede seguir siendo efectivo.

En el caso de la caída de presión del etanol, esta disminuye ya que con un incremento de $\mathrm{t}_{1}$ disminuye el valor de la MLDT [ecuación (8)], incrementándose tanto el área de transferencia de calor necesaria (A) [ec. (9)] como el número de tubos requeridos $\left(\mathrm{N}_{\mathrm{t}}\right)$ [ec. (10)]. Lo anterior provoca que el área de flujo del etanol dentro de los tubos $\left(a_{e t}\right)$ aumente [ec. $\left.(, 21)\right]$ para entonces disminuir la velocidad del etanol dentro de los tubos $\left(\mathrm{v}_{\mathrm{et}}\right)$ [ec. (22)] y, con ello, disminuya tanto la caída de presión debido al paso del etanol por los tubos del enfriador $\left(\Delta \mathrm{p}_{\mathrm{t}}\right)$ [ec. (50)] como la caída de presión debido al paso del etanol un paso hacia otro $\left(\Delta \mathrm{p}_{\mathrm{n}}\right)$ [ec. (51)], para obtener al final una disminución de la caída de presión total del etanol. Vale destacar que aún para un valor límite de $\mathrm{t}_{1}$ de $10^{\circ} \mathrm{C}$ la caída de presión total del etanol obtenida para esta temperatura (8 402,39 Pa) no supera la caída de presión permisible establecida por el proceso (10 $000 \mathrm{~Pa})$.

Con respecto al incremento que experimenta la potencia de cada motor acoplado a los ventiladores con un aumento de $t_{1}$, esto se debe a que la densidad del aire se reduce a medida que $t_{1}$ aumenta [ec. (5)], lo cual incrementa la velocidad del aire en el ventilador ( $\mathrm{v}_{\text {aire }}$ [ [ec. (61)], la presión que deberán desarrollar los ventiladores $\left(\mathrm{P}_{\mathrm{v}}\right)$ [ec. (62)], y el caudal volumétrico de aire de cada ventilador (qaire) [ec. (63)], por lo que también aumentará la potencia del motor de cada ventilador $\left(\mathrm{P}_{\mathrm{m}}\right)$ [ec. (64)]. Es decir, mientras más caliente sea el aire de entrada, mayor potencia tendrá el motor acoplado al ventilador. 
De esta manera se sugiere operar a la menor temperatura posible del aire de entrada, para que la caída de presión total del etanol se mantenga dentro del valor establecido y también para que la potencia del motor acoplado al ventilador tenga el menor valor posible y, con ello, el equipo diseñado consuma la menor cantidad de energía eléctrica posible.

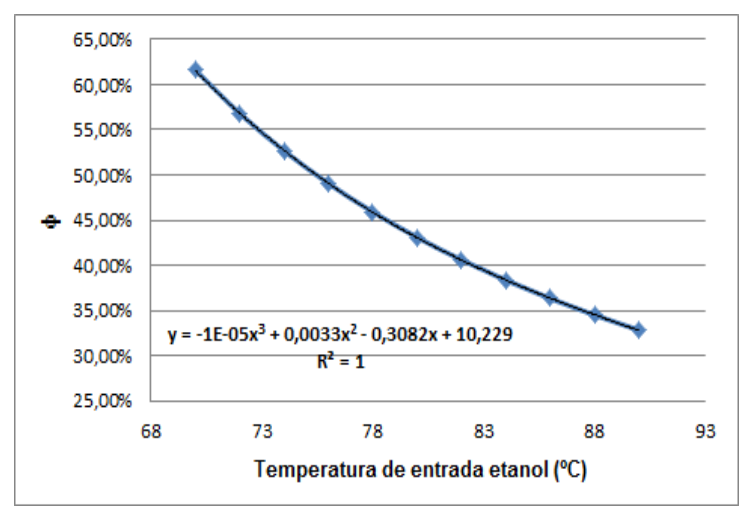

(a)

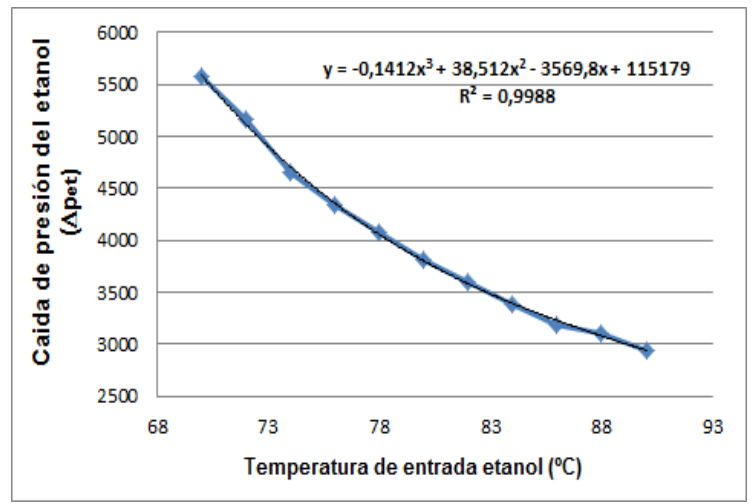

(b)

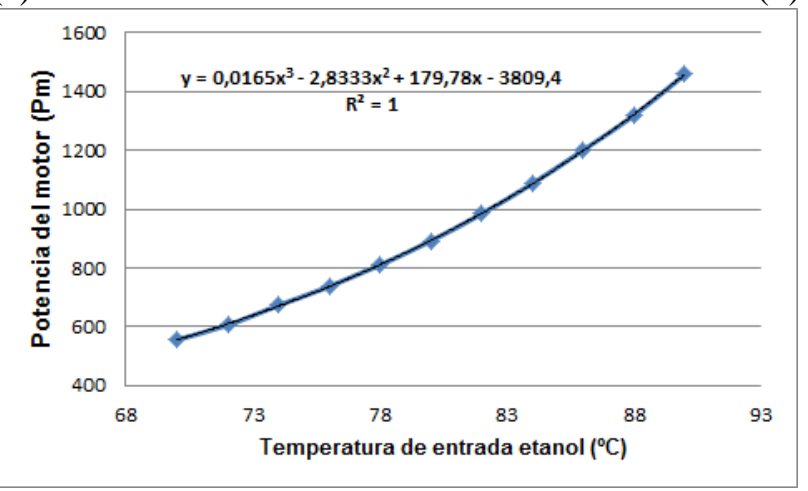

(c)

Figura 4. Influencia del incremento de la temperatura de alimentación del etanol sobre tres parámetros de diseño del equipo. (a) Relación porcentual entre el área proyectada por los ventiladores y el área frontal $(\phi)$. (b) Caída de presión total del etanol $\left(\Delta \mathrm{p}_{\mathrm{et}}\right)$. (c) Potencia del motor acoplado a cada ventilador $\left(\mathrm{P}_{\mathrm{m}}\right)$.

Fuente: Elaboración propia

De acuerdo con la Figura 4, el incremento de la temperatura de alimentación del etanol $\left(\mathrm{T}_{1}\right)$ reduce tanto el parámetro $\phi$ como la caída de presión total del etanol, e incrementa la potencia del motor acoplado al ventilador. En el caso de la Figura 4a), se puede observar que a partir de los $84{ }^{\circ} \mathrm{C}$ de temperatura el parámetro $\phi$ comienza a presentar un valor por debajo del $40 \%$, lo cual indica que el diseño del equipo comienza a ser inefectivo a partir de esta temperatura.

Con relación a la Figura 4c), el aumento de $\mathrm{T}_{1}$ incrementa la potencia del motor debido a que aumenta la carga de calor intercambiado $(\mathrm{Q})$ [ec. (2)] y, con ello, el caudal másico de aire $\left(\mathrm{m}_{\text {aire }}\right)$ requerido para cumplir con el servicio de transferencia de calor demandado [ec. (4)]. El incremento de $\mathrm{m}_{\text {aire }}$ aumenta la velocidad del aire en el ventilador ( $\mathrm{v}_{\text {aire }}$ ) [ec. (61)], y esto incrementa, a su vez, la presión que deberá ser desarrollada por los ventiladores $\left(\mathrm{P}_{\mathrm{v}}\right)$ [ec. (62)], lo cual posibilita que aumente finalmente la potencia del motor acoplado a cada ventilador $\left(\mathrm{P}_{\mathrm{m}}\right)$ [ec. (64)].

De acuerdo con los resultados obtenidos, se recomienda que la temperatura de alimentación del etanol al equipo se mantenga, en lo posible, en el valor establecido por el proceso $\left(80^{\circ} \mathrm{C}\right)$ para el parámetro $\phi$ se mantenga superior al $40 \%$, y la potencia del motor no se incremente hasta alcanzar valores prohibidamente elevados. 


\section{CONCLUSIONES}

El enfriador por aire diseñado tendrá ancho de 1,64 m, un número de tubos requeridos igual a 60 , una cantidad de tubos igual a 60, un número de filas de tubos de 6 , y un número de tubos por fila de 10.

El etanol fluirá por dentro del tubos bajo régimen turbulento, ya que el número de Reynolds calculado para este fluido ( $\left(\mathrm{Re}_{\mathrm{et}}\right)$ es superior a 10000 (16 271,60), mientras que se obtuvo una eficiencia de la aleta de 0,8776 .

Se obtuvo un valor del coeficiente pelicular de transferencia de calor de $716,62 \mathrm{~W} / \mathrm{m}^{2} . \mathrm{K}$ y $715,51 \mathrm{~W} / \mathrm{m}^{2} . \mathrm{K}$ para el etanol y el aire, respectivamente, para obtener un resultado del coeficiente global de transferencia de calor calculado de $327,59 \mathrm{~W} / \mathrm{m}^{2} . \mathrm{K}$, el cual es $45,4 \%$ menor que el asumido ( $\left.600 \mathrm{~W} / \mathrm{m}^{2} . \mathrm{K}\right)$.

Se obtuvo un valor del parámetro relación porcentual entre el área proyectada por los ventiladores y el área frontal $(\phi)$ de $43,13 \%$, el cual es superior al $40 \%$ por lo que el diseño realizado es efectivo, obteniéndose así una distribución uniforme en el enfriador.

La caída de presión total del etanol por los tubos del enfriador alcanzó un valor de 3 785,64 Pa, el cual es inferior a la caída de presión permisible establecida para esta corriente (10 $000 \mathrm{~Pa})$.

Cada uno de los dos motores utilizados para hacer funcionar los dos ventiladores consume una potencia de $897 \mathrm{~W}$.

Se recomienda operar a la menor temperatura posible para el aire de entrada, con el fin de reducir la potencia del motor acoplado al ventilador, y para que el parámetro $\phi$ se mantenga por encima del $40 \%$.

Se aconseja que la temperatura de alimentación del etanol al equipo se conserve en el valor establecido por el proceso $\left(80^{\circ} \mathrm{C}\right.$ ) para que el parámetro $\phi$ se mantenga superior al $40 \%$, y no se incremente la potencia del motor hasta alcanzar un valor muy elevado.

\section{REFERENCIAS}

Amercool. (2007). Basics of Air Cooled Heat Exchangers. Tulsa, Oklahoma, USA: Amercool Manufacturing Inc.

Boes, S. (2017). Improve Air-Cooled Heat Exchanger Performance. Chemical Engineering Progress, January 2017, $1-7$.

Cao, E. (2010). Heat transfer in process engineering. New York: The McGraw-Hill.

Elmetenani, S., Yousfi, M. L., Merabeti, L., Belgroun, Z., and Chikouche, A. (2011). Investigation of an evaporative air cooler using solar energy under Algerian climate. Energy Procedia, 6, 573-582. doi:10.1016/j.egypro.2011.05.066.

Giammaruti, R. (2004). Performance Improvement to Existing Air-Cooled Heat Exchangers. Cooling Technology Institute Annual Conference, Houston, Texas, USA.

GPSA. (2004). Air-Cooled Exchangers. Engineering Data Book (12th ed.). Tulsa, Oklahoma, USA: Gas Processors Suppliers Association.

Hudson. (2007). The Basics of Air-Cooled Heat Exchangers. Texas, USA: Hudson Products Corporation. 
Kröger, D. G. (2004). Air-Cooled Heat Exchangers and Cooling Towers. Thermal-flow Performance Evaluation and Design. (Doctor of Engineering Thesis), University of Stellenbosch, Stellenbosch, South Africa.

Laval, A. (2018). Intercambiadores de calor por aire. Lund, Suecia: Alfa Laval.

Lu, Y., Guan, Z., Hooman, K., and Parulekar, P. S. (2017). An Investigation on Cooling Performance of Air-Cooled Heat Exchangers Used in Coal Seam Gas Production. Heat Transfer Engineering, 38 (11-12), 1073-1088. doi: 10.1080/01457632.2016.1217039.

Mijeev, M. A., y Mijeeva, I. M. (1979). Fundamentos de termo-transferencia. Moscú: Editorial Mir.

Mukherjee, R. (1997). Effectively Design Air-Cooled Heat Exchangers. Chemical Engineering Progress, February 1997, 26-47.

Mustafa, A. T., and Jasim, H. S. (2018). Performance evaluation of a developed evaporative air cooler with solar irradiance effect. International Journal of Engineering \& Technology, 7 (4), 4785-4791. doi: 10.14419/ijet.v7i4.14033.

Perry, R. H., and Green, D. W. (2008). Perry's Chemical Engineers' Handbook (8th ed.). New York, USA: McGrawHill.

Thulukkanam, K. (2013). Heat Exchanger Design Handbook (2nd ed.). Boca Ratón, FL, USA: CRC Press.

Yousif, H., and Hussain, A. (2012). Optimum Design of Evaporative Air Cooler. Journal of Kerbala University, 10 (2), 287-299.

\section{SEMBLANZA DE LOS AUTORES}

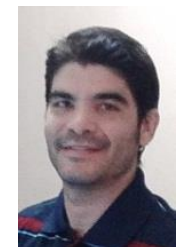

Amaury Pérez Sánchez: Obtuvo el grado de Ingeniero Químico en la Universidad de Camagüey, Cuba, donde actualmente es profesor instructor e investigador auxiliar. En estos momentos se encuentra estudiando un Máster en Biotecnología en el centro de Ingeniería Genética y Biotecnología de Camagüey. Trabaja en líneas de investigación vinculadas fundamentalmente con el diseño de equipos de transferencia de calor y masa, simulación y optimización de procesos y operaciones de la industria química mediante el empleo de simuladores, y la evaluación técnico-económica de procesos y plantas biotecnológicas.

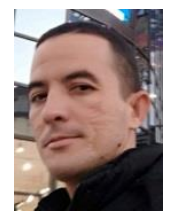

Yosdany Hernández González: Obtuvo el grado de Ingeniero Mecánico en la Universidad de Camagüey, Cuba en el año 2007. Actualmente se desempeña profesionalmente como Especialista A en la Empresa de Ingeniería y Proyectos Azucareros UEB Centro Este, de la ciudad de Camagüey, Cuba, específicamente en la especialidad termoenergética. Trabaja en líneas de investigación relacionadas fundamentalmente con la evaluación de sistemas termoenergéticos de fábricas de azúcar, así como también la modelación, simulación y diseño en 3D de operaciones, procesos y sistemas de la industria azucarera, mediante la aplicación de software tales como CADWork ${ }^{\circledR}$, CAESARII $^{\circledR}$ y SOLIDWORKS ${ }^{\circledR}$.

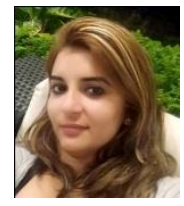

Elizabeth Ranero González. Obtuvo el grado de Ingeniero Químico en la Universidad de Camagüey, Cuba. Se desempeña profesionalmente como Profesor Instructor en la Universidad de Camagüey, Cuba. Su área de investigación abarca el diseño y evaluación de equipos y procesos de transferencia de calor y masa, evaluación termodinámica de procesos químicos, y la simulación de procesos y sistemas biotecnológicos.

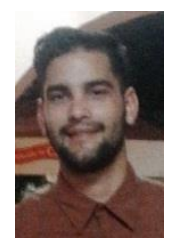

Eddy Javier Pérez Sánchez: Obtuvo el grado de Ingeniero Químico en la Universidad de Camagüey, Cuba. Se desempeña profesionalmente en la Empresa de Servicios Automotores S.A., en el Departamento de Dirección Comercial. Trabaja en líneas de investigación relacionadas con el diseño, evaluación y/o operación de equipos de transferencia de calor y masa, asi como también la simulación de procesos petroquímicos y biotecnológicos. 